\title{
Phase Sensitive Signal Processing using Semiconductor Optical Amplifiers
}

\author{
Andrew D. Ellis ${ }^{1}$, Stylianos Sygletos ${ }^{2}$ \\ ${ }^{1}$ Aston Institute of Photonic Technology, Aston University, Birmingham B4 7ET, England, \\ ${ }^{2}$ Tyndall National Institute, University College Cork, Ireland, \\ Authore-mail address: andrew.ellis@aston.ac.uk
}

\begin{abstract}
This paper examines recent progress in the use of semiconductor optical amplifiers for phase sensitive signal processing functions, a discussion of the world's first multi-wavelength regenerative wavelength conversion using semiconductor optical amplifiers for BPSK signals. OCIS codes: 070.4340 Nonlinear optical signal processing, 190.4380 Four-wave mixing, 060.4510 Optical coms
\end{abstract}

\section{Introduction}

An impending capacity crunch has been widely discussed recently, arising from the inability of a single optical fiber strand to continue to support the exponentially growing traffic demands [1]. Many solutions have been proposed including novel optical amplifiers, installation of new fibers to allow spatial multiplexing and the re-emergence of optical regeneration [2]. Of these only optical regeneration offers a significant increase in the overall capacity of a fully populated link without significant increases in cost or power consumption. Conventional (Silica based) highly nonlinear fiber (HNLF) with high SBS threshold has proven effective in enabling many of the essential features of a future all optical regenerator, including

- Operation with phase encoded signals, to ensure compliance with coherently detected signal formats [3,4]

- Multi-wavelength operation, to ensure cost competitiveness with optical amplifiers [5]

- Simultaneous amplitude and phase regeneration, to give the prospects of regeneration of QAM signals [6]

- Quantum limited performance, to maximize performance benefits [7]

Despite the excellent fiber performance [eg 8] these reports typically employ at least one high power (Watt class) optical amplifier, reducing the prospects for compact and low energy consumption devices. Several alternative material systems exist for the production of nonlinear devices based on four wave mixing processes including periodically poled lithium niobate [9], photonic crystal [10] and/or bismuth fiber [11, 12], calcogenide planar waveguides [13], polymer clad silicon waveguides [14] and amorphous silicon [15], unfortunately when practical configurations are considered such devices either require higher pump powers than for the equivalent function in HNLF, or have power handling limitations such as two photon absorption or the Staebler-Wronski effect. In contrast to the materials above operating on variations of the Kerr effect, nonlinearity in SOA's is mediated by carrier excitation enabling signal processing with significantly lower optical power levels [16]. SOAs have been previously demonstrated in many FWM based devices including wavelength conversion [17], clock recovery [18],optical sampling [19] and optical regeneration [20], but very little work has been performed on phase sensitive applications $[21,22]$. In this paper, we report on the first ever multi-channel regeneration in a semiconductor optical amplifier [22]. This is achieved in a fully "black box", fiber in - fiber out configuration, operating with phase modulated data. The system summarized here employs semiconductor devices for all active functions, opening the possibility of a fully integrated optical subsystem. Optical power requirements are modest $(<4 \mathrm{~mW}$ total launched power at each SOA) eliminating the need for additional optical amplification and the overall power consumption will dominated by the SOA power supply which will be greatly reduced by future cooler less optical amplifiers [23]. The presentation will summarize this work, and consider opportunities for optical regeneration in coherent systems.

\section{Experimental Setup}

A typical experimental implementation is shown in Fig.1. In this case two independent DPSK transmitters are passed through a phase modulator driven by a $3.3 \mathrm{GHz}$ sinusoidal wave to introduce the phase distortion on both channels for and a moderate output power (14dBm) Erbium doped fiber amplifier (EDFA) amplified the at the input to the regenerator. A free running continuous wave laser was combined with the signal and injected into a $1 \mathrm{~mm}$ polarization insensitive bulk SOA (24dB gain, $12 \mathrm{dBm}$ output power) device where FWM was used to achieve simultaneous carrier extraction (see fig. 1b) of the two incoming DPSK signals [24] with a total launch power of less than $4 \mathrm{~mW}$ and a short optical path which minimized thermally induced phase variations. The generated carriers were regenerated using optically injection locked discrete mode lasers (less than -35dBm input power). A wavelength selective switch (WSS) combined the two incoming DPSK signals with appropriate power levels and the 
three local pumps and directed them into the second SOA. The spectrum is shown in Fig 1(c) at the output of the first WSS. The second SOA, used for phase sensitive was also $1 \mathrm{~mm}$ long but and had a total input power of less than $5.6 \mathrm{dBm}$. Through degenerate four-wave mixing, the two DPSK signals interacted with the free running laser and their corresponding regenerated pump wave in the second SOA creating degenerate idlers that beat with them. However, due to competing parametric and non-parametric gain of the saturated device the contrast ratio (as a function of pump phase) of the signals is greatly reduced. This is not the case for the non-degenerate FWM components which presented a significant phase sensitive gain, providing an efficient phase squeezing. Fig. 1(d) shows the optical spectrums at the output of the second SOA when the PSA was simultaneously locked at the maximum (black) or minimum (red) phase sensitive gain for both channels. Independent gain contrasts of $\sim 20 \mathrm{~dB}$ were achieved for both channels. Fig. 1(e) shows the output when the channels were locked at different phase states.
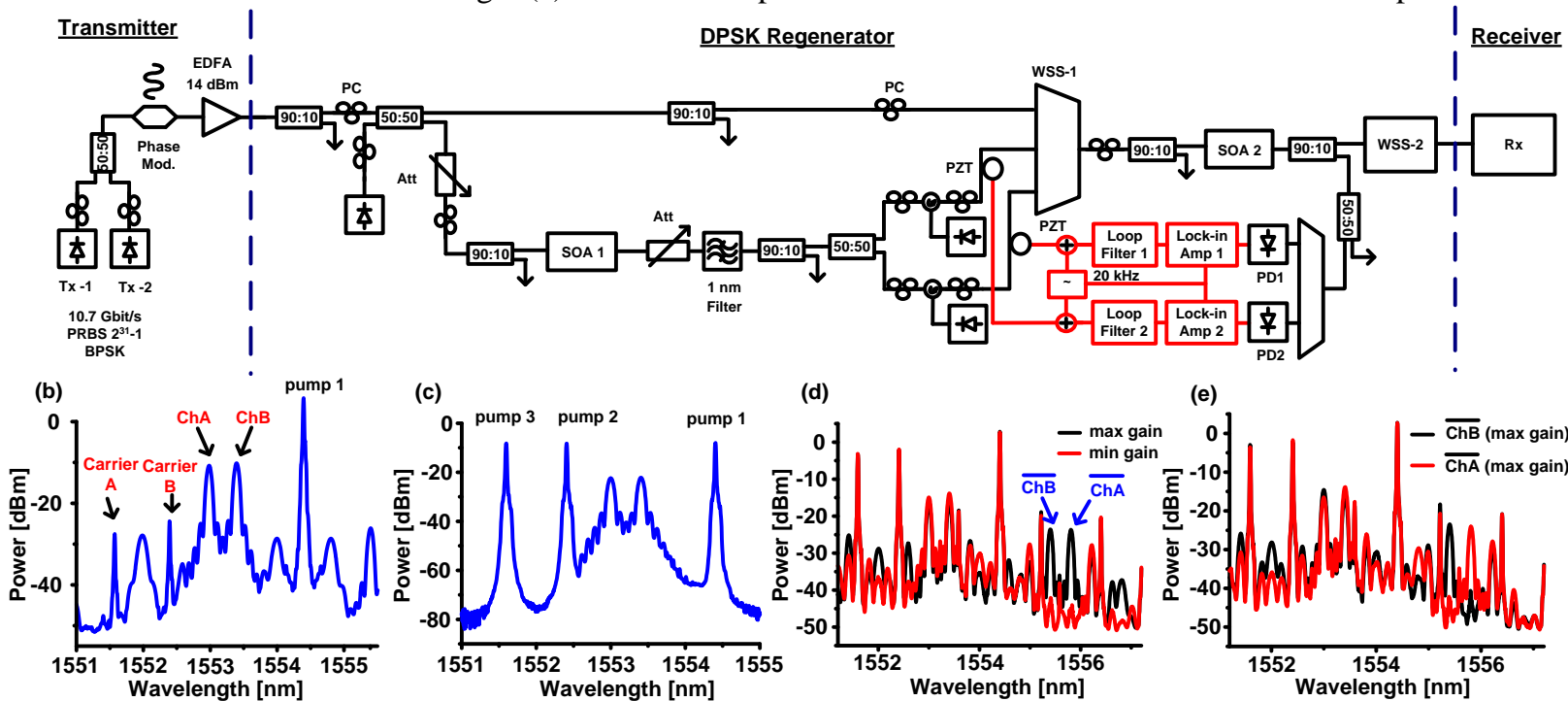

(d)

(e)

Fig. 1 (a) Experimental setup of the two channel PSA. (b) Spectrum after the carrier extraction stage (output of SOA 1). (c) Spectrum at the output of the WSS. (d) Spectrum at the output of SOA-2 when both channels are locked to the maximum or minimum phase sensitive gain state of the PSA. (e) Spectrum at the output of the SOA 2 when the channels are locked at different phase sensitive gain states of the PSA.

The performance of the regenerator has been investigated as a function of the relative power levels between the pumps and the signals entering the regenerative stage of the PSA, i.e. the SOA-2 device, controlled using the first WSS. The solid lines in Fig. 2a show the show receiver sensitivity penalty at the regenerator output for the two channels as a function of the ratio of power between the pumps to signals. Less than $1 \mathrm{~dB}$ penalty is observed for a power ratio between 6 and $12 \mathrm{~dB}$ bounded signal to noise ratio and data induced carrier depletion mechanisms in the SOA. Dotted lines show the phase regeneration under the same conditions when the two input channels have been subjected to a periodic phase distortion (3dB penalty at the PSA input). Sensitivity improvements approaching $2 \mathrm{~dB}$ are observed over the same operating range. We also notice that the channel closest to the free running pump slightly outperforms the other. This may be attributed to the higher FWM efficiency for closely spaced signals.

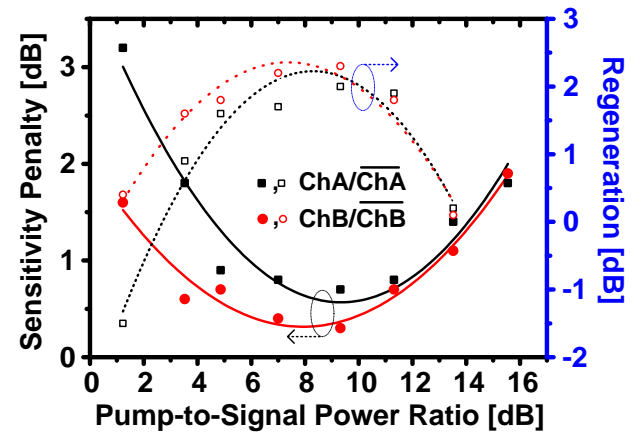

(a)

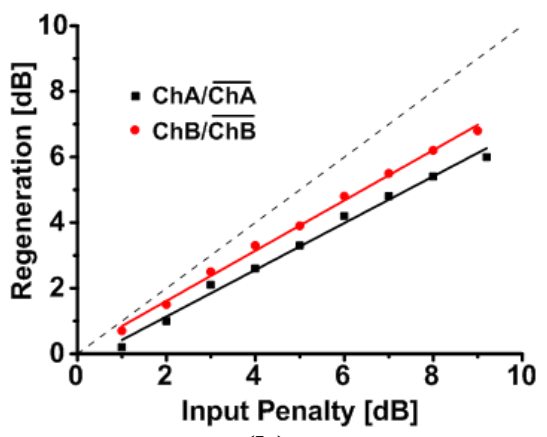

(b)

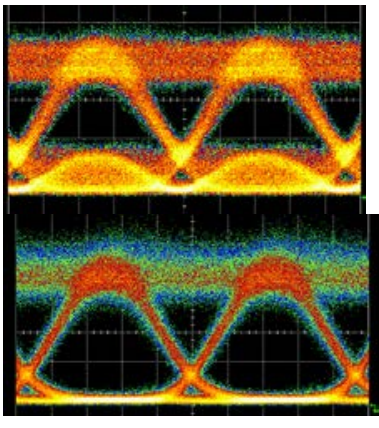

(c)

Fig. 2 (a) Performance of PSA as a function of the pump-to signal power ratio in terms of sensitivity penalty with undistorted input (solid lines) and regeneration for a $3 \mathrm{~dB}$ degradation at the regenerator input (dotted line). (b) Receiver sensitivity improvement as a function of the input penalty induced by periodic phase distortion. (c) Eye diagrams for ChA when both input signals have been degraded by $9 \mathrm{~dB}$. 
Fig. 2b depicts the improvement in the received sensitivity of the two DPSK signals, measured at the output of the PSA, versus the penalty introduced at the input. More than $5 \mathrm{~dB}$ regeneration is offered by the scheme when the applied distortion results in an input penalty of 9dB. Although, such levels off improvement wouldn't be expected when a random phase noise is considered, these results confirm the steepness in the nonlinear phase transfer function of the phase squeezing scheme. The differentially demodulated eye diagrams measured using a single ended photodiode shown in Fig. 2c (with 9dB input penalty) clearly show the corresponding phase restoration.

\section{Conclusion}

In this paper, we have reported the characterization of the world's first fiber in-fiber out dual wavelength direct phase regeneration using semiconductor optical amplifiers. The regenerative capabilities for phase distorted signals are within a $\mathrm{dB}$ of ideal performance (complete regeneration of all degradation with no output penalty) and we anticipate that the development and integration of future cooler less SOAs will enable the generation of compact, low power consumption multi-channel all optical regenerators.

This work was supported in part by Science Foundation Ireland under grant number 06/IN/I969 and the authors would like to thank R.J.Manning and R.Webb for useful discussions and the loan of SOAs used in this experiment.

\section{References}

1. A.R Chraplyvy, “The coming capacity crunch”, ECOC 2009 Plenary paper, (2009).

2 A.D.Ellis, "Current trends in optical communications", proc OSA conference on Frontiers in Optics, paper FiO5.4, (2012).

3 R. Slavík, F. Parmigiani, J. Kakande, C. Lundström, M. Sjödin, P. A. Andrekson, R. Weerasuriya, S. Sygletos, A. D. Ellis, L. GrünerNielsen, D. Jakobsen, S.. Herstrøm, R. Phelan, J. O’Gorman, A. Bogris, D. Syvridis, S. Dasgupta, P. Petropoulos, D. J. Richardson., “Alloptical phase and amplitude regenerator for next-generation telecommunications systems”, Nature Photonics Vol. 4, No. 10, pp690-695 (2010).

4 J.Kakande, R.Slavík, F.Parmigiani, A.Bogris, D.Syvridis, L.Grüner-Nielsen, R.Phelan, P.Petropoulos, D.J.Richardson, “Multilevel quantization of optical phase in a novel coherent parametric mixer architecture”, Nature Photonics, Vol. 5, pp.748, (2011).

5 S. Sygletos, P. Frascella, S.K. Ibrahim, L. Grüner-Nielsen, R. Phelan, J. O’Gorman, A.D. Ellis, “A Practical Phase Sensitive Amplification Scheme for Two Channel Phase Regeneration”, Optics Express, Vol. 19, No. 26, pp. B938-B945 (2011).

6 T. Roethlingshoefer et al., "Multilevel Amplitude and Phase Regeneration in a Nonlinear Amplifying Loop Mirror with a Phase-Sensitive Amplifier”, Proc ECOC 2012, paper Th.1.A.3, (2012).

7 Z. Tong, C. Lundström, P. A. Andrekson, C. J. McKinstrie, M. Karlsson, D. J. Blessing, E. Tipsuwannakul, B. J. Puttnam, H. Toda, and L. Grüner-Nielsen, “Towards ultrasensitive optical links enabled by low-noise phasesensitive amplifiers," Nature Photonics 5(7), 430-436 (2011).

8 Lars Grüner-Nielsen, Søren Herstrøm, Sonali Dasgupta, David Richardson, Dan Jakobsen, Carl Lundström, Peter A. Andrekson, Martin E. V. Pedersen, and Bera Pálsdóttir "Silica-Based Highly Nonlinear Fibers with a High SBS Threshold” Proc IEEE Photonics Society Winter Topical meeting; 2011, Paper MD4.2; (2011).

9 M.H. Chou, I Brener, M.M. Fejer, E.E. Chaban, S.B. Christman, "1.5-mm-band wavelength conversion based on cascaded second-order nonlinearity in LiNbO3 waveguides', Photonics Technology Letters, Vol. 11, pp. 653-655, (1999).

10 P.A.Andersen; T.Tokle; Yan Geng; C.Peucheret; P.Jeppesen; , "Wavelength conversion of a 40-Gb/s RZ-DPSK signal using four-wave mixing in a dispersion-flattened highly nonlinear photonic crystal fiber," Photonics Technology Letters,Vol.17,no.9, pp.1908-1910, (2005).

11 N.Sugimoto, T.Nagashima, T.Hasegawa, S.Ohara, "Bismuth-based optical fiber with nonlinear coefficient of 1360 W/sup -1/ km/sup -1/," Proc OFC 2004, vol.2, (2004).

12 K. K. Chow, K. Kikuchi, T. Nagashima, T. Hasegawa, S. Ohara, and N. Sugimoto, "Four-wave mixing based widely tunable wavelength conversion using 1-m dispersion-shifted bismuth-oxide photonic crystal fiber," Optics Express, Vol. 15, pp.15418-15423 (2007).

13 M.D., Pelusi, V.G.Ta'eed, F. Libin E.Magi, M.R.E.Lamont, S.Madden, D.-Y. Choi; D.A.P. Bulla, B.Luther-Davies, B.J.Eggleton, "Applications of Highly-Nonlinear Chalcogenide Glass Devices Tailored for High-Speed All-Optical Signal Processing,", Journal of Selected Topics in Quantum Electronics, Vol.14, no.3, pp.529-539, (2008).

14 J.Leuthold,W.Freude, C.Koos, T.Vallaitis, J,-M. Brosi, S.Bogatscher, P.Dumon, R.Baets, M.L.Scimeca, I.Biaggio, F.Diederich, "Siliconorganic hybrid (SOH) - A platform for ultrafast optics," Proc. ECOC '09, (2009).

15 K. Narayanan and S.F. Preble, "Optical nonlinearities in hydrogenated-amorphous silicon waveguides," Optics Express Vol. 18, pp.89989005, (2010).

16 D'Ottavi, A.; Iannone, A.; Mecozzi, A.; Scotti, S.; Spano, P.; Dall'Ara, R.; Eckner, J.; Guekos, G.; , "Efficiency and noise performance of wavelength converters based on FWM in semiconductor optical amplifiers," Photonics Technology Letters, Vol.7, no.4, pp357-359, (1995).

17 A.E.Kelly, A.D.Ellis, D.Nesset, R.Kashyap, D.G.Moodie, "100 Gbit/s wavelength conversion using FWM in an MQW semiconductor optical amplifier", Electronics Letters, Vol.34, No.20, pp 1955, (1998).

18 O. Kamatani, S.Kawanishi, "Ultrahigh-speed clock recovery with phase lock loop based on four-wave mixing in a traveling-wave laser diode amplifier," Journal of Lightwave Technology, Vol.14, no.8, pp.1757-1767, (1996).

19 T. Morioka, H. Takara, S. Kawanishi, K. Uchiyama, and M. Saruwatari, "Polarization independent all-optical demultiplexing up to 200Gbit/s using four wave mixing in a semiconductor laser amplifier,” Electronics Letters, Vol. 32, pp. 840-841, (1996).

20 C. Porzi, A. Bogoni, G. Contestabile, "Regeneration of DPSK Signals in a Saturated SOA", Photonics Technology Letters, Vol. 24, No. 18, pp1597-1599, (2012).

21 R.P. Webb, J.M. Dailey, R.J. Manning, and A.D. Ellis, "Phase discrimination and simultaneous frequency conversion of the orthogonal components of an optical signal by four-wave mixing in an SOA," Optics Express, Vol. 19, pp. 20015-20022 (2011).

22 S. Sygletos, M. Power, F. C. Garcia Gunning, R. P. Webb, R. J. Manning, A. D. Ellis, "Simultaneous Dual Channel Phase Regeneration in SOAs", ECOC 2012, Tu1A2, (2012).

23 S.J.Sweeney, "New semiconductor approaches to energy efficient integrated photonics", in Proc ICOOPMA12 International Conference on Optical, Optoelectronic and Photonic Materials and Applications 2012, Nara, Japan, Paper 1C1-1, (2012).

24 R. Weerasuriya et al. "Generation of frequency symmetric signals from a BPSK input for phase sensitive amplification,” in Proc. OFC 2010 paper OWT6 (2010). 